\title{
Interventricular Septal Dissection in a Patient with an Old Myocardial Infarction
}

\author{
Akio NaKata, Satoshi HiRota, Hiroshi Tsuji and Eisuke Takazakura
}

\begin{abstract}
We observed an unusual case of interventricular septal wall dissection in a patient with a prior myocardial infarction. Echocardiography, magnetic resonance imaging, and left ventriculography revealed separation of the right-side and left-side walls of the interventricular septum with an accessory chamber between the two walls. Morphologic findings were consistent with interventricular septal dissection.
\end{abstract}

(Internal Medicine 35: 33-35, 1996)

Key words: pseudo-false aneurysm, accessory chamber, echocardiography, magnetic resonance imaging, left ventriculography

\section{Introduction}

Left ventricular pseudoaneurysm is a rare complication that results from rupture of the left ventricular free wall in the setting of an acute myocardial infarction, surgery, trauma or endocarditis (1-3). The walls of pseudoaneurysms are composed of hematoma and pericardium, and lack myocardial elements. Cases in which myocardial elements are contained in the aneurysmal wall have been classified as pseudo-false aneurysms (4). Cases of pseudo-false aneurysms involving the left ventricular free wall, but not the interventricular septum have been described. Here we report a pseudo-false aneurysm-like lesion involving the interventricular septal wall due to septal dissection in a patient who had suffered a myocardial infarction 12 years earlier and who was admitted to our hospital with congestive heart failure.

\section{Case Report}

A 53-year-old Japanese man, who suffered from an acute anteroseptal myocardial infarction in 1979, had been treated conservatively at another hospital. Diabetes mellitus wàs also diagnosed at that time. He was treated with glibenclamide, but he discontinued taking the oral medication after discharge. In 1982, he was treated at our hospital for diabetes mellitus, but he discontinued treatment in February 1991. In August 1991, he was admitted to our hospital with worsening dyspnea.

On admission, physical examination revealed peripheral cyanosis and crackles in the entire lung field. He had a heart rate of 120 beats/min and his blood pressure was $174 / 80 \mathrm{mmHg}$. Chest radiographs revealed cardiomegaly and pulmonary congestion. An electrocardiogram revealed sinus tachycardia and evidence of a prior anteroseptal myocardial infarction. Laboratory examination demonstrated an elevated white cell count $(10,500 / \mu \mathrm{l})$, lactate dehydrogenase $(605 \mathrm{IU} / l)$, creatinine phosphokinase $(335 \mathrm{IU} / \mathrm{l})$, fasting blood sugar $(260 \mathrm{mg} / \mathrm{dl})$ and hemoglobin $\mathrm{A}_{1 \mathrm{c}}(12.7 \%)$. Analysis of arterial blood gases in room air showed a pH of 7.39, a $\mathrm{PCO}_{2}$ of 43.4 Torr, and a $\mathrm{PO}_{2}$ of 38.5 Torr. The congestive heart failure was treated with oxygen and diuretics.

Two-dimensional echocardiography showed that the interventricular septum was separated into right-side and left-side walls, that enclosed an accessory chamber. This accessory chamber communicated with the left ventricle via the orifice of the left-side septal wall (Fig. 1). Doppler examination showed no septal perforation.

Magnetic resonance imaging also revealed two interventricular septal walls and the division of the left ventricle into two chambers (Fig. 2).

Cardiac catheterization was performed. Coronary cineangiography showed a total occlusion of the left anterior descending artery at proximal sites, which was believed to be responsible for the old myocardial infarction. Left ventriculography showed the left-side septal wall of the left ventricle that formed the accessory chamber. The two chambers communicated with each other through a defect in the left-side septal wall, but no communication was observed between the left and right ventricular chambers (Fig. 3).

From the Department of Internal Medicine, Kurobe Municipal Hospital, Kurobe

Received for publication April 10, 1995; Accepted for publication September 18, 1995

Reprint requests should be addressed to Dr. Akio Nakata, the Department of Internal Medicine, Kurobe Municipal Hospital, 1108-1 Mikkaichi, Kurobe 938 


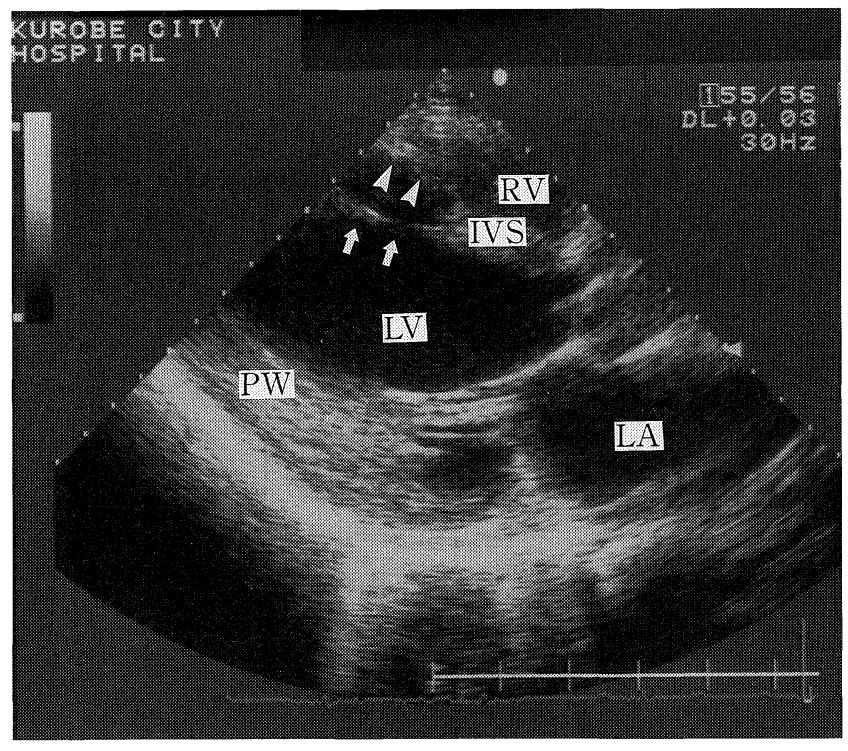

A

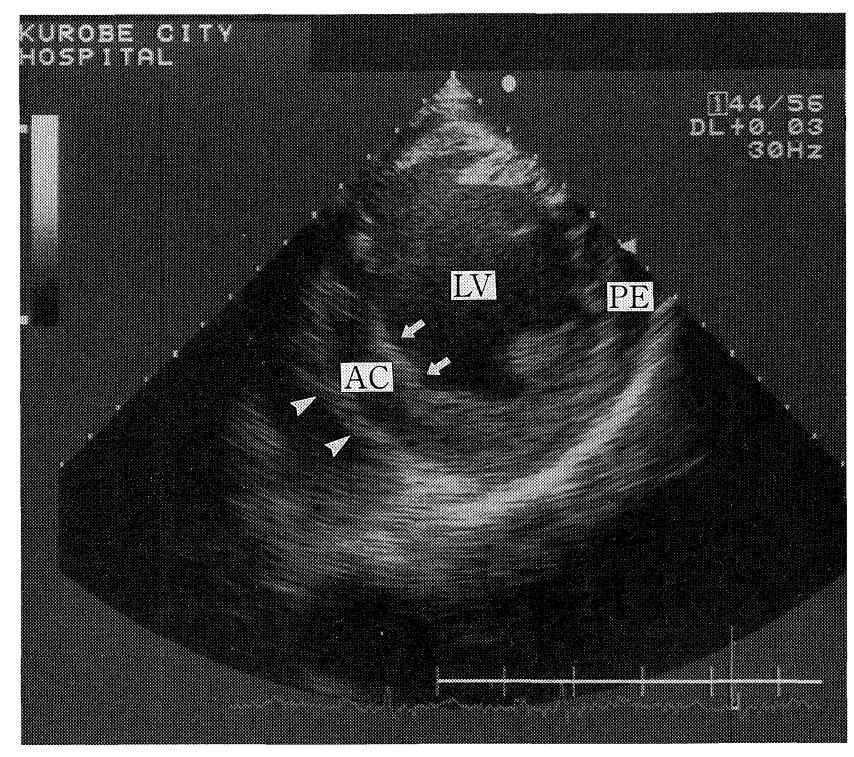

$\mathrm{B}$

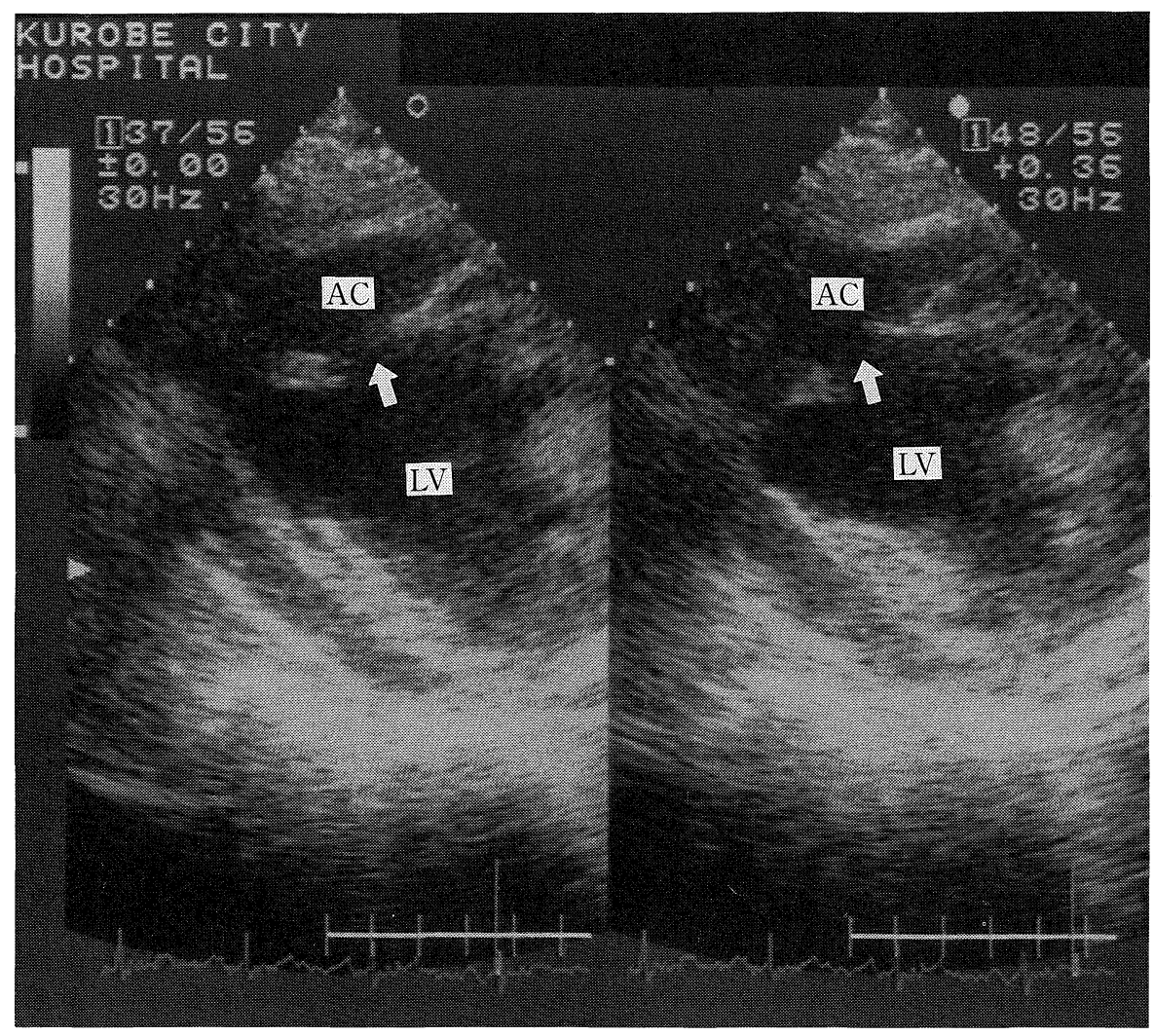

$\mathrm{C}$

Figure 1. Two-dimensional echocardiography. Parasternal long-axis view (Panel A) and transesophageal short-axis view (Panel B) demonstrating separation of the interventricular septum into left-side (arrows) and right-side (arrowheads) layers. Parasternal short-axis views in systole (right) and in diastole (left) show the orifice of the left-side septal wall (arrow) connecting the left ventricular chamber to the accessory chamber formed between the right-side and the left-side septal layers (Panel C).AC: accessory chamber, IVS: interventricular septum, LA: left atrium, LV: left ventricle, PE: pericardial effusion, PW: posterior wall, $R V$ : right ventricle. 


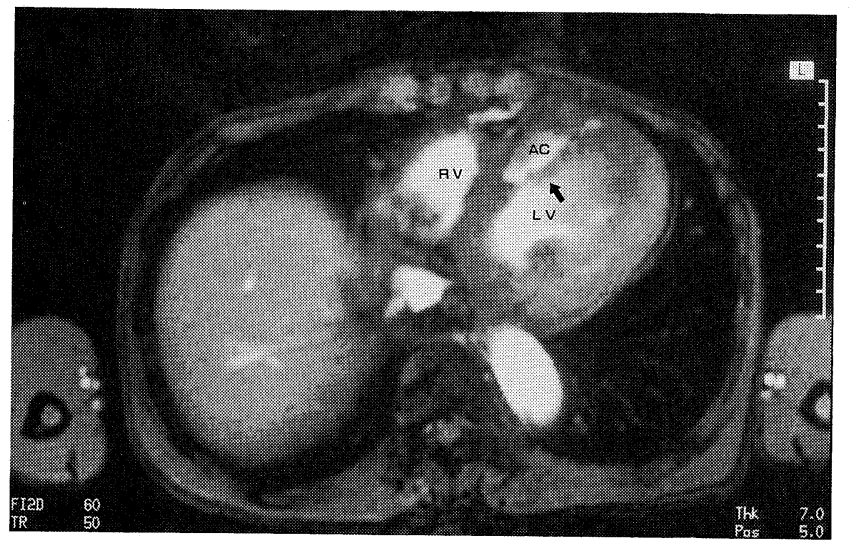

Figure 2. Transverse gradient echo magnetic resonance image obtained using a 1.0 Tesla magnet. Arrows indicate the leftside septal wall. Abbreviations are the same as in Fig. 1.

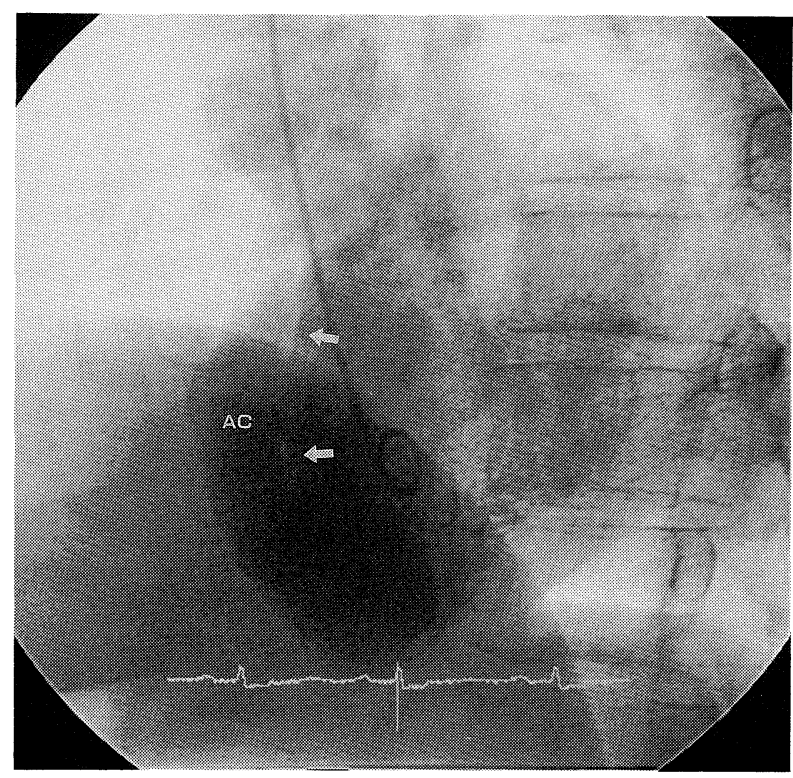

Figure 3. Left ventriculogram from the left anterior oblique projection. Arrows indicate the left-side septal wall. Abbreviations are the same as in Fig. 1.

Based on these findings, we made the diagnosis of interventricular septal dissection following myocardial infarction. Surgical repair was recommended, but the patient refused and he was treated conservatively. Three years later (August 1994), the patient was admitted to our hospital with a recurrence of congestive heart failure. Echocardiography, magnetic reso- nance imaging, and left ventriculography performed at that time showed no changes in the morphology of the interventricular septum.

\section{Discussion}

Echocardiography, magnetic resonance imaging and left ventriculography showed a longitudinal separation of the interventricular septal wall in the present case. Two-dimensional echocardiography has shown that pseudoaneurysms are distinguished from true aneurysms by the presence of a relatively narrow orifice compared with the maximal parallel internal diameter of the cavity $(5,6)$. Stewart et al described pseudoaneurysm-like lesions in which myocardial fiber is present in the aneurysmal walls, which they classified as pseudo-false aneurysms. They detected a pseudo-false aneurysm in the left ventricular free wall (4). Although no pathological examination was performed in the present study, imaging studies suggested the presence of a similar lesion in the interventricular septal wall. We characterized this lesion as an "interventricular septal dissection" based on the morphologic findings. We speculate that an incomplete tear in the necrotized interventricular septum led to longitudinal laceration without causing perforation. There have been no previous reports of interventricular septal dissection. In the absence of an interventricular septal rupture, the presence of interventricular septal dissection may not deteriorate hemodynamics and may be asymptomatic. The present patient has survived 3 years following the diagnosis of interventricular septal dissection, and 15 years following myocardial infarction without surgical treatment. Thus, the natural history of interventricular septal dissection may be benign, but further studies are necessary.

\section{References}

1) Gueron M, Wanderman KL, Hirsch M, Borman J. Pseudoaneurysm of the left ventricle after myocardial infarction: A curable form of myocardial rupture. J Thorac Cardiovasc Surg 69: 736, 1975.

2) Spellberg RD, O'Reilly RJ. Pseudoaneurysm of the left ventricle following mitral valve replacement. Chest 62: 115, 1972.

3) Jamshidi A, Berry RW. Left ventricular pseudoaneurysm secondary to cardiac stab wound. Successful repair in a thirteen year old girl. Am J Cardiol 16: 601, 1965.

4) Stewart S, Huddle R, Stuard I, Schreiner BF, DeWeese JA. False aneurysm and pseudo-false aneurysm of the left ventricle: etiology, pathology, diagnosis, and operative management. Ann Thorac Surg 31: 259, 1981.

5) Catherwood E, Mintz GS, Kotler MN, Parry WR, Segal BL. Twodimensional echocardiographic recognition of left ventricular pseudoaneurysm. Circulation 62: 294, 1980.

6) Gatewood RP Jr, Nanda NC. Differentiation of left ventricular pseudoaneurysm from true aneurysm with two dimensional echocardiography. Am J Cardiol 46: 869, 1980. 